\title{
Distribution, Temporal Stability and Appropriateness of Therapy of Patients With COPD in the UK in Relation to GOLD 2019
}

\author{
David M.G. Halpin ${ }^{\mathrm{a}, \mathrm{b}, *}$, Hilda J.I. de Jong ${ }^{\mathrm{b}}$, Victoria Carter ${ }^{\mathrm{b}}$, Derek Skinner ${ }^{\mathrm{b}}$, David Price ${ }^{\mathrm{b}}$ \\ a University of Exeter Medical School, Exeter, UK \\ ${ }^{\mathrm{b}}$ Observational and Pragmatic Research Institute Pte Ltd (OPRI), Singapore
}

\section{A R T I C L E I N F O}

\section{Article history:}

Received 1 May 2019

Received in revised form 10 June 2019

Accepted 16 July 2019

Available online 24 July 2019

\section{Keywords:}

COPD

GOLD

Clinical practice

Pharmacotherapy

Comorbidities

\begin{abstract}
A B S T R A C T
Background: The 2019 Global Initiative for Chronic Obstructive Lung Disease (GOLD) report made recommendations for the assessment, initial and subsequent treatment chronic obstructive pulmonary disease (COPD) based on biomarkers, including blood eosinophil counts.

Methods: We evaluated the distribution of UK COPD patients initiating maintenance therapy and established patients by GOLD group, the prevalence of comorbidities and appropriateness of therapy using electronic patient records from the Optimum Patient Care Research Database (OPCRD). Changes in effective GOLD group, therapy and exacerbation rates over the next 2 years were analysed.

Findings: 11,409 established COPD patients and 699 starting therapy were studied. $44.3 \%, 25.7 \%, 13.8 \%$ \& $16 \cdot 2 \%$ of established COPD patients and $45 \cdot 2 \%, 28.5 \%, 15.7 \%$ \& $10.6 \%$ initiating therapy were in GOLD groups $A, B, C \& D$ respectively.

The overall proportion in each GOLD group was similar after 2 years but there was substantial movement of patients between groups. Diabetes and cardiovascular disease were the most common comorbidities in all groups in both cohorts.

LAMA monotherapy was the commonest initial therapy in all GOLD groups. In both cohorts there was overtreatment with escalation, de-escalation or switching in nearly 50\% during follow-up.

In both cohorts, exacerbation rates were highest in group $\mathrm{D}$ and appeared higher in over-treated patients. Interpretation: Most patients are not at risk of exacerbations and co-morbidities are common. Many patients change effective GOLD group and therapy over time. Prescribing is not in accordance with guideline recommendations and many patients still appear over treated.
\end{abstract}

(C) 2019 Published by Elsevier Ltd. This is an open access article under the CC BY-NC-ND license.

(http://creativecommons.org/licenses/by-nc-nd/4.0/)

\section{Introduction}

In 2017 the Global Obstructive Lung Disease initiative (GOLD) published new recommendations on the assessment of COPD [1] separating the degree of spirometric impairment from the four GOLD groups A to D which classify patients on the basis of the level of symptoms and the risk of exacerbations, assessed now solely by the number of exacerbations in the previous year. The 2019 GOLD Report clarifies previous recommendations that the groups are solely used as the basis for the choice of initial therapy [2]. Many clinicians had interpreted the recommendations as if they applied to patients at any stage of their management, although this was not what was stated or intended and previous studies on the appropriateness of therapy have studied populations that were already receiving maintenance therapy rather than examining initial treatment choices [3-6].

\footnotetext{
* Corresponding author at: University of Exeter Medical School, Exeter, UK.

E-mail address: d.halpin@nhs.net (D.M.G. Halpin).
}

These studies have shown a higher use of ICS and under-prescription of bronchodilators than expected based on the recommendations [3,7-11]; however, their interpretation is limited by the fact some patients may have responded well to therapy and reduced the level of their symptoms, had fewer exacerbations or both, resulting in erroneous conclusions about the appropriateness of their therapy.

We applied the 2017 assessment scheme to patients with COPD under the care of General Practitioners in the United Kingdom (UK) to determine the distribution of patients and whether it identified groups with different outcomes such as exacerbation rate. As in studies of the distribution of patients according to previous GOLD recommendations, we examined the distribution into the four groups among established patients with COPD (cohort 1), but importantly we have also investigated the distribution of patients initiating maintenance therapy (cohort 2), who are the true target of the GOLD assessment scheme. We also examined the distribution of spirometric impairment and comorbidities among patients in each group in both established patients and those initiating maintenance therapy. We examined the 


\section{Research in Context}

\section{Evidence Before This Study}

In 2019 GOLD published updated recommendations on the management of COPD focussing on the initial and subsequent use of inhaled therapy. Previous studies have compared the use of therapy in practice with previous GOLD recommendations and have examined outcomes such as exacerbation rates in people categorised into the four GOLD groups A to D. However, these studies have almost exclusively been in people already on maintenance therapy when it is impossible to determine if therapy is appropriate or not as it may have changed the level of symptoms of exacerbation rates leading to false conclusions.

\section{Added Value of This Study}

To our knowledge, this is the first study to compare prescribing patterns in a large cohort of unselected patients with COPD to the GOLD 2019 recommendations for initial and subsequent therapy whilst also examining the changes in breathlessness and exacerbation rates over time and their relationship to the appropriateness of the therapy. Many patients in groups A $\&$ B who are at low risk of exacerbations appeared overtreated, particularly with inhaled corticosteroids, but the exacerbation rates in these patients were higher than those appropriately treated suggesting additional clinical features may have influenced the prescription of therapy. The study also shows for the first time in a large primary care cohort the marked changes in patients' level of symptoms and number of exacerbations over time leading to movement between GOLD groups.

\section{Implications of All the Available Evidence}

The GOLD assessment scheme does predict patients at higher risk of exacerbation and patients' level of symptoms and number of exacerbations changes over time, either spontaneously or as a result of therapy. GOLD groups should not be used to assess the appropriateness of established therapy this should be based on the level of continuing breathlessness and number of exacerbations, taking into account the blood eosinophil count. Compared to the GOLD recommendations there is significant overtreatment, but other factors may guide individual treatment choices and in some cases these may be appropriate. Further work to characterise these would assist the development of future treatment recommendations.

appropriateness of the initial inhaled therapy in those initiating maintenance therapy, and the appropriateness of the maintenance inhaled therapy in the established COPD patients with reference to the GOLD 2019 recommendations. We determined whether patients appeared to have changed groups, what the annual exacerbation rates were and whether these were related to the appropriateness of therapy.

\section{Methods}

\subsection{Data Source}

A population-based cohort study was carried out using the continuous electronic health record database, Optimum Patient Care Research Database (OPCRD) which contains anonymised routine, patient-level diagnostic, clinical and prescribing information on approximately 5.8 million patients from over 700 primary care centres across the UK (http://opcrd.co.uk/). OPCRD has received a favourable opinion from the NHS Health Research Authority for anonymous research use (REC reference: 15/EM/0150). Governance is provided and study protocol was approved by the Anonymised Data Ethics and Protocols Transparency (ADEPT) committee (ADEPT0918), an independent body of experts and regulators commissioned by the Respiratory Effectiveness Group (REG, http://www.effectivenessevaluation.org/). The study protocol was registered with the European Union electronic Register of Post-Authorization studies (EUPAS25136).

\subsection{Study Population}

All patients with established COPD (had at least one diagnostic Read code (Quality Outcomes Framework (QOF) defined) for COPD before the index date (ID)) who were continuously registered at a general practice between January 1, 2013 and December 31, 2016 were eligible for inclusion in the analysis. To be coded as having COPD patients had to have had spirometry. Cohort 1 comprised patients with established COPD, irrespective of maintenance treatment for COPD. In this cohort the ID was defined as January 1, 2014. Cohort 2 comprised established COPD patients who initiated maintenance treatment in the year 2014. The date of initiating maintenance treatment for COPD was defined as the ID, the date at which the patient received their first prescription of long-acting beta agonists (LABA), long-acting muscarinic antagonists (LAMA), inhaled corticosteroids (ICS), or combination therapies of LABA, LAMA and ICS (LAMA/LABA, ICS/LABA, ICS/LAMA, LABA/LAMA/ICS) in the year 2014. Patients in cohort 2 were not included in cohort 1 . For further details of the data requirements and data extracted from OPCRD see the appendix.

The primary outcome in each cohort was the change of GOLD groups over a 2-year period. The classification of each patient by GOLD group in 2016 was compared with their GOLD group in 2014. Additional outcomes were the appropriateness of the therapy with reference to GOLD 2019 in patients in cohort 1 based on their breathlessness (mMRC score), exacerbation frequency and blood eosinophil count (for details of the criteria used see appendix, table S1), and the appropriateness of the initial therapy in cohort 2 (see appendix, table S2). Patients were classified as under-, appropriately or over-treated on the ID. The number of patients who appeared over-treated who had a diagnosis of current asthma was also calculated. Patients were further categorised as having escalation, de-escalation, switching or no change in their therapy between 2014 and 2016 (for details of the criteria used see appendix, table S3).

In both cohorts the number of exacerbations and pneumonia events for each patient were calculated over 2-year follow-up. Exacerbations were identified using the criteria described in the appendix. Pneumonia events were defined by all relevant diagnostic read codes. Read codes that were recorded within 28 days of each other were considered to belong to the same pneumonia event.

\subsection{Statistical Analysis}

Kappa statistics were used to examine changes in GOLD groups over the 2-year period, and were interpreted according to the classification system proposed by Landis and Koch [12]. We evaluated the relationship between GOLD group and exacerbation rates within each cohort, using a negative binomial regression model allowing over-dispersion with GOLD group A as the reference group. A subgroup analysis based on appropriateness of maintenance therapy (under-, appropriate and over-treatment) according to GOLD 2019 was also conducted for exacerbations. Since the majority of patients had no pneumonia during follow-up, a zero-inflated negative binomial regression model was used to examine the association between the GOLD groups and pneumonia, and findings were reported as rate ratio (RR)s (95\% CIs). The change in maintenance treatment over two years were compared between the GOLD groups within cohort 2, using a multinominal logistic regression model with findings reported as relative risk ratios (RRRs) with the corresponding 95\% CIs. In the unconditional 
models, findings were adjusted for age and sex. All other potential confounders did not change the association. Potential confounders were included in the final model if they independently changed the estimated effect for the GOLD groups by $\geq 10 \%$. Potential confounders, as measured at baseline, included smoking and inhaled corticosteroid use. Because of the low percentage of missing data in the study (see Tables 1 and 2), no imputation method was used. All analyses were conducted using SAS V9.3 (SAS Institute Inc., Cary, N.C.).

\subsection{Complementary Analysis}

We expected the prevalence of comorbidities to be higher in patients with established COPD (cohort 1) compared with those initiating maintenance therapy (cohort 2); however, this was not the case in our preliminary analysis [13]. We therefore examined the prevalence of comorbid conditions in a general population to determine whether the overall prevalence of comorbidities was higher in patients with established COPD, reflecting the systemic effects of the disease, including at an early stage, or whether it is simply a feature of an older population group (for details see appendix).

\section{Results}

11,409 patients were identified as established COPD patients forming cohort 1 and 699 patients started maintenance therapy for the first time during 2014 forming cohort 2 (Fig. 1). Among established patients $44.3 \%$ had the characteristics of GOLD group A, 25.7\% group $B, 13.8 \%$ group $C$ and $16.2 \%$ group $D$ (Table 1 ). In patients initiating maintenance therapy, $45.2 \%$ were in GOLD group A, $28.5 \%$ in group $B, 15.7 \%$ in group $C$ and $10.6 \%$ in group $D$ (Table 2 ).

\subsection{Baseline Characteristics}

The mean age of established patients was approximately 70 years, with patients in group B being a little older than those in the other groups (Table 1). There was more variation in the age of patients starting maintenance therapy with those in groups $B$ \& D being slightly older (Table 2). In established patients, men outnumbered women in groups $A$ \& B but the numbers were approximately equal in groups C \& $D$, whereas men accounted for approximately $60 \%$ of patients in all groups initiating therapy. The prevalence of current smoking was about 30\% in established COPD patients with no difference between groups, but it was higher in patients initiating therapy (at 45-47\%). Approximately $17 \%$ of established patients in groups A \& B and $22-24 \%$ in groups C \& D had a diagnosis of current asthma (Table 1). A lower proportion of patients in each GOLD group initiating therapy had asthma (Table 2).

The distribution of $\mathrm{FEV}_{1}$ impairment was similar among established patients (Table 1), although there were more GOLD stage 3 and 4 patients in group $\mathrm{D}$. In patients initiating maintenance therapy there were very few GOLD stage 4 patients in group A and more stage 3 and 4 patients in groups $C$ and $D$. The mean mMRC score for patients initiating therapy in groups $A$ and $C$ was $0.7-0.8$ and $2.3-2.5$ in patients in groups B \& D. There was no difference between mMRC scores in established patients in groups $\mathrm{A}$ and $\mathrm{C}$ or between groups $\mathrm{B}$ and $\mathrm{D}$, but the mean scores were higher in established COPD patients (cohort 1 ) in groups B \& D compared with those initiating therapy (cohort 2) (Tables 1 \& 2).

The mean exacerbation rates in the 12 months prior to ID were similar in both cohorts, with mean rates in patients in groups $A$ and $B$ between $0.3-0.4$ per year. Over $60 \%$ of patients in these groups had not had an exacerbation in the previous year. In groups $C$ and $D$ of both cohorts the mean rate was approximately $2.6-3.3$ per year.

Table 1

Baseline characteristics of established COPD patients.

\begin{tabular}{|c|c|c|c|c|c|}
\hline \multirow[t]{2}{*}{ Baseline characteristics } & \multirow{2}{*}{$\frac{\text { Group A }}{N=5053}$} & \multirow{2}{*}{$\frac{\text { Group B }}{\mathrm{N}=2938}$} & \multirow{2}{*}{$\frac{\text { Group C }}{N=1574}$} & \multirow{2}{*}{$\frac{\text { Group D }}{\mathrm{N}=1844}$} & \multirow{2}{*}{$\frac{\text { Overall }}{N=11409}$} \\
\hline & & & & & \\
\hline$\%$ of patients in group & $44 \cdot 3$ & 25.7 & $13 \cdot 8$ & $16 \cdot 2$ & - \\
\hline Mean age $(\mathrm{y})(\mathrm{SD})$ & $70.4(9.5)$ & $72 \cdot 1(9 \cdot 6)$ & $69.9(9.6)$ & $70.4(9.5)$ & $70 \cdot 8(9.6)$ \\
\hline Men \% & $56 \cdot 9$ & 55.0 & 51.9 & 47.9 & $54 \cdot 3$ \\
\hline Non-missing, $\mathrm{n}(\%)$ & 5049 (99.9) & 2937 (99.9) & $1574(100)$ & $1842(99.9)$ & $11,402(99 \cdot 9)$ \\
\hline Mean BMI (SD) & $27.3(5 \cdot 3)$ & $28.7(6.7)$ & $27.3(5.8)$ & $28.3(6.8)$ & $27.8(6.0)$ \\
\hline Non-missing, $\mathrm{n}(\%)$ & $5026(99.5)$ & $2925(99.6)$ & $1572(99.9)$ & $1837(99.6)$ & $11,360(99.6)$ \\
\hline Current smokers n (\%) & $1571(31 \cdot 3)$ & $906(31.0)$ & $534(34.0)$ & $572(31 \cdot 1)$ & $3583(31.5)$ \\
\hline Ex-smokers n (\%) & $2886(57.4)$ & $1713(58.6)$ & $847(53.9)$ & $1095(59.6)$ & $6541(57.6)$ \\
\hline Non-smokers n (\%) & $569(11 \cdot 3)$ & $306(10.4)$ & $191(12 \cdot 2)$ & $170(9 \cdot 3)$ & $1236(10.9)$ \\
\hline Asthma (\%) & $877(17.4)$ & $522(17.8)$ & $379(24 \cdot 1)$ & $409(22 \cdot 2)$ & $2187(19.2)$ \\
\hline Non-missing, $\mathrm{n}(\%)$ & $5017(99 \cdot 3)$ & $2915(99.2)$ & $1558(98.9)$ & $1834(99.5)$ & $11,324(99 \cdot 3)$ \\
\hline Mean $\mathrm{FEV}_{1} \%$ predicted(SD) & $67.5(21 \cdot 3)$ & $59.3(22.5)$ & $64 \cdot 2(32 \cdot 3)$ & $55 \cdot 4(22 \cdot 1)$ & $63.0(24.0)$ \\
\hline Gold $1: \geq 80 \%$ & $1268(26 \cdot 2)$ & $443(15 \cdot 7)$ & $312(20 \cdot 8)$ & $238(13.5)$ & $2261(20 \cdot 7)$ \\
\hline Gold 2: $50-79 \%$ & $2697(55.8)$ & $1443(51 \cdot 3)$ & $781(52 \cdot 1)$ & $791(44 \cdot 8)$ & $5712(52 \cdot 3)$ \\
\hline Gold 3: $30-49 \%$ & $697(14.4)$ & $701(24 \cdot 9)$ & $313(20.9)$ & $532(30 \cdot 1)$ & $2243(20 \cdot 6)$ \\
\hline Gold 4: $<30 \%$ & $174(3.6)$ & $228(8 \cdot 1)$ & $93(6 \cdot 2)$ & $206(11.6)$ & $701(6.4)$ \\
\hline Mean exacerbations/y (SD) & $0.3(0.5)$ & $0.4(0.5)$ & $2 \cdot 8(1.3)$ & $3.3(1.8)$ & $1.2(1.6)$ \\
\hline Exacerbations/y in previous year & $3495(69.2)$ & $1819(61.9)$ & $0(0)$ & $0(0)$ & $5314(46 \cdot 6)$ \\
\hline & $1558(30.8)$ & $1119(38 \cdot 1)$ & $0(0)$ & $0(0)$ & $2677(23.5)$ \\
\hline & $0(0)$ & $0(0)$ & $1574(100 \cdot 0)$ & $1844(100 \cdot 0)$ & $3418(29.9)$ \\
\hline Mean mMRC (SD) & $0.7(0.5)$ & $2.4(0.6)$ & $0.8(0.4)$ & $2.5(0.6)$ & $1.4(1.0)$ \\
\hline Non-missing, $\mathrm{n}(\%)$ & $4675(92.5)$ & $2802(95.4)$ & $1506(95 \cdot 7)$ & $1776(96 \cdot 3)$ & $10,759(94 \cdot 3)$ \\
\hline Mean Eosinophil count $\left(10^{9} / \mathrm{L}\right)(\mathrm{SD})$ & $0.2(0.2)$ & $0.2(0.2)$ & $0.3(0.3)$ & $0.3(0.2)$ & $0.3(0.2)$ \\
\hline Eosinophil count $<0.3010^{9} / \mathrm{L}$ & $3152(67.4)$ & $1911(68.2)$ & $986(65.5)$ & $1136(64.0)$ & $7185(66.8)$ \\
\hline Eosinophil count $\geq 0.3010^{9} / \mathrm{L}$ & $1523(32 \cdot 6)$ & $891(31.8)$ & $520(34.5)$ & $640(36 \cdot 0)$ & $3574(33 \cdot 2)$ \\
\hline \multicolumn{6}{|c|}{ Maintenance therapy in the year before index date, $\mathrm{n}(\%)$} \\
\hline None & $796(15.8)$ & $190(6 \cdot 5)$ & $68(4 \cdot 3)$ & $19(1 \cdot 0)$ & $1073(9.4)$ \\
\hline LAMA only & $1259(24.9)$ & $764(26 \cdot 0)$ & $368(23.4)$ & $376(20.4)$ & $2767(24 \cdot 2)$ \\
\hline LABA only & $215(4 \cdot 2)$ & $86(2.9)$ & $47(3.0)$ & $23(1.3)$ & $371(3.2)$ \\
\hline ICS only & $537(10 \cdot 6)$ & $163(5 \cdot 6)$ & $145(9 \cdot 2)$ & $63(3.4)$ & $908(8.0)$ \\
\hline ICS + LABA & $1448(28.7)$ & $882(30.0)$ & $530(33.7)$ & $609(33.0)$ & $3469(30.4)$ \\
\hline ICS + LAMA & $51(1.0)$ & $39(1 \cdot 3)$ & $18(1 \cdot 1)$ & $13(0.7)$ & $121(1 \cdot 1)$ \\
\hline LAMA + LABA & $55(1 \cdot 1)$ & $44(1.5)$ & $15(1.0)$ & $8(0.4)$ & $122(1 \cdot 1)$ \\
\hline ICS + LABA + LAMA & $692(13.7)$ & $770(26 \cdot 2)$ & $383(24.3)$ & $733(39.8)$ & $2578(22.6)$ \\
\hline
\end{tabular}


Table 2

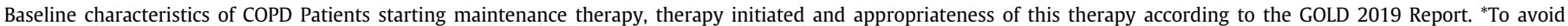
unintentional disclosure and to preserve confidentiality, cell counts $<5$ are reported as $<5$.

\begin{tabular}{|c|c|c|c|c|c|}
\hline \multirow[t]{2}{*}{ Baseline characteristics } & \multirow{2}{*}{$\frac{\text { Group A }}{\mathrm{N}=316}$} & \multirow{2}{*}{$\frac{\text { Group B }}{\mathrm{N}=199}$} & \multirow{2}{*}{$\frac{\text { Group C }}{\mathrm{N}=110}$} & \multirow{2}{*}{$\begin{array}{l}\text { Group D } \\
\mathrm{N}=74\end{array}$} & \multirow{2}{*}{$\begin{array}{l}\text { Overall } \\
\mathrm{N}=699\end{array}$} \\
\hline & & & & & \\
\hline$\%$ of patients in group & $45 \cdot 2$ & 28.5 & $15 \cdot 7$ & $10 \cdot 6$ & - \\
\hline Mean age $(\mathrm{y})(\mathrm{SD})$ & $67.9(9.9)$ & $69.8(11.7)$ & $68.8(9.6)$ & $70 \cdot 6(10 \cdot 3)$ & $68.9(10.5)$ \\
\hline Men \% & 59.5 & 58.3 & 58.2 & 59.5 & 58.9 \\
\hline Non-missing, \% & $314(99.4)$ & $199(100)$ & $109(99.1)$ & $74(100)$ & $696(99.6)$ \\
\hline Mean BMI (SD) & $27.3(5.9)$ & $28 \cdot 2(6 \cdot 3)$ & $27 \cdot 2(5 \cdot 1)$ & $27 \cdot 6(6 \cdot 2)$ & $27.6(5.9)$ \\
\hline Non-missing, \% & $315(99.7)$ & $199(100)$ & $110(100)$ & $74(100)$ & $698(99.9)$ \\
\hline Current smokers n (\%) & $147(46 \cdot 7)$ & $89(44.7)$ & $50(45 \cdot 5)$ & $35(47.3)$ & $315(45 \cdot 1)$ \\
\hline Ex-smokers n (\%) & $143(45.4)$ & $93(46 \cdot 7)$ & $48(43.6)$ & $37(50 \cdot 0)$ & $327(46.9)$ \\
\hline Non-smokers n (\%)(\%) & $25(7.9)$ & $17(8.6)$ & $12(10 \cdot 9)$ & $<5^{*}$ & $56(8.0)$ \\
\hline Asthma (\%) & $37(11.7)$ & $20(10 \cdot 1)$ & $18(16.4)$ & $6(8 \cdot 1)$ & $81(11 \cdot 6)$ \\
\hline Non-missing, \% & $314(99.4)$ & $199(100)$ & $109(99.1)$ & $73(97.3)$ & $695(99.4)$ \\
\hline Mean FEV ${ }_{1}(\%$ pred)(SD) & $66.6(18.7)$ & $57.6(20.3)$ & $64 \cdot 3(19.4)$ & $59.1(22.9)$ & $62.9(20 \cdot 1)$ \\
\hline Gold $1: \geq 80 \%$ & $64(21.0)$ & $22(11.6)$ & $19(18.4)$ & $12(16 \cdot 9)$ & $117(17.5)$ \\
\hline Gold 2: $50-79 \%$ & $187(61 \cdot 3)$ & $99(52.4)$ & $63(61 \cdot 2)$ & $33(46 \cdot 5)$ & $382(57.2)$ \\
\hline Gold 3: $30-49 \%$ & $50(16.4)$ & $56(29 \cdot 6)$ & $17(16 \cdot 5)$ & $19(26 \cdot 8)$ & $142(21 \cdot 3)$ \\
\hline Gold $4:<30 \%$ & $<5$ & $12(6.4)$ & $<5$ & $7(9 \cdot 8)$ & $27(4.0)$ \\
\hline Mean exacerbations/y (SD) & $0.3(0.5)$ & $0.4(0.5)$ & $2 \cdot 6(1.0)$ & $2.7(1.4)$ & $1.4(0.9)$ \\
\hline Exacerbations/y in previous year 0 & $225(71.2)$ & $124(62 \cdot 3)$ & $0(0)$ & $0(0)$ & $349(49.9)$ \\
\hline 1 & $91(28.8)$ & $75(37.7)$ & $0(0)$ & $0(0)$ & $166(23.7)$ \\
\hline$\geq 2$ & $0(0)$ & $0(0)$ & $110(100 \cdot 0)$ & $74(100 \cdot 0)$ & $184(26 \cdot 3)$ \\
\hline Mean mMRC $(\overline{S D})$ & $0.7(0.5)$ & $2.3(0.6)$ & $0.8(0.4)$ & $2.3(0.5)$ & $2.4(0.6)$ \\
\hline Non-missing, \% & $291(92 \cdot 1)$ & $187(93.7)$ & $102(92.7)$ & $68(91.9)$ & $648(92.7)$ \\
\hline Mean Eosinophil count $\left(10^{9} / \mathrm{L}\right)(\mathrm{SD})$ & $0.2(0.2)$ & $0.2(0.2)$ & $0.3(0.2)$ & $0.2(0 \cdot 1)$ & $0.3(0.2)$ \\
\hline Eosinophil count $<0.30 \times 10^{9} / \mathrm{L}$ & $194(66 \cdot 7)$ & $118(63.1)$ & $60(58.8)$ & $49(72 \cdot 1)$ & $421(65 \cdot 0)$ \\
\hline Eosinophil count $\geq 0.30 \times 10^{9} / \mathrm{L}$ & $97(33 \cdot 3)$ & $69(36 \cdot 9)$ & $42(41 \cdot 2)$ & $19(27.9)$ & $227(35 \cdot 0)$ \\
\hline \multicolumn{6}{|l|}{ Therapy initiated n (\%) } \\
\hline LAMA only & $155(49 \cdot 1)$ & $100(50 \cdot 3)$ & $44(40 \cdot 0)$ & $35(47 \cdot 3)$ & $334(47 \cdot 8)$ \\
\hline LABA only & $19(6 \cdot 0)$ & $7(3 \cdot 5)$ & $6(5.4)$ & $<5$ & $36(5 \cdot 1)$ \\
\hline ICS only & $50(15 \cdot 8)$ & $18(9.0)$ & $16(15 \cdot 6)$ & $<5$ & $87(12 \cdot 5)$ \\
\hline $\mathrm{ICS}+\mathrm{LABA}$ & $79(25.0)$ & $59(29.7)$ & $39(35 \cdot 5)$ & $20(27 \cdot 0)$ & $197(28 \cdot 2)$ \\
\hline ICS + LAMA & $<5$ & $0(0)$ & $0(0)$ & $<5$ & $5(0.7)$ \\
\hline LAMA + LABA & $<5)$ & $<5$ & $<5$ & $0(0)$ & $7(1.0)$ \\
\hline ICS + LABA + LAMA & $8(2.5)$ & $11(5 \cdot 5)$ & $<5$ & $10(13.5)$ & $33(4.7)$ \\
\hline \multicolumn{6}{|l|}{ Treatment according to Gold 2019 Report (\%) } \\
\hline Under-treatment & $0(0)$ & $0(0)$ & $22(20 \cdot 0)$ & $7(9.5)$ & - \\
\hline Appropriate treatment & $174(55 \cdot 1)$ & $107(53 \cdot 8)$ & $44(40 \cdot 0)$ & $57(77.0)$ & - \\
\hline Over-treatment & $142(44.9)$ & $92(46 \cdot 2)$ & $44(40 \cdot 0)$ & $10(13 \cdot 5)$ & - \\
\hline Number of overtreated patients with coexistent asthma & $28(19.7)$ & $18(19 \cdot 6)$ & $10(22.7)$ & $<5$ & - \\
\hline
\end{tabular}

Mean eosinophil counts were similar in patients in all GOLD groups and across cohorts, with approximately two thirds of the patients having counts below $300 \times 10^{9} / \mathrm{L}$.

Over half of established patients were on therapy containing ICS and a quarter were on LAMA alone, with no differences between the GOLD groups. More established patients in group D were on triple therapy (39.8\%) than those in groups B (26.2\%) and C (24.3\%), and triple therapy was only used in $13.7 \%$ of patients in group $A$ (Table 1 ).

In patients initiating therapy, LAMA monotherapy was the commonest treatment in all groups, with ICS containing therapy used in less than $50 \%$ of the patients. Very few patients in groups A, B \& C were started on triple therapy, but it was the first maintenance therapy used in $13.5 \%$ of patients in group D.

\subsection{Change of GOLD Groups Over 2 years}

In both cohorts, the overall proportion of patients in each GOLD group was similar in 2014 and 2016 (cohort 1: Kappa: 0.33, 95\% CI: 0.32-0.35; cohort 2: Kappa: 0.03, 95\% CI: $-0.01-0.07$ ), but there had been substantial movement of individual patients (Fig. 2). In both cohorts, over half of the patients in groups A and B at baseline stayed in these groups, but over $20 \%$ of patients in group B at baseline in both cohorts moved into group A (i.e. became less symptomatic). Over the two years $41.2 \%$ of established COPD patients categorised as meeting the criteria for group D in 2014 moved to groups A or B on the basis of having had fewer exacerbations. 52.7\% COPD patients in group D started on maintenance therapy in 2014 moved to groups A or B, with only $36.5 \%$ staying in group D over 2 years.

\subsection{Appropriateness of Therapy}

In established COPD patients there appeared to be substantial overtreatment of patients with blood eosinophil counts $<100$ and those with higher eosinophil counts with $\mathrm{mMRC}<2$ and fewer than 2 exacerbations in the previous year (Table 3 ). The medications prescribed to patients judged as over-treated are shown in Appendix table S4. In the majority of cases over-treatment was due to the inappropriate use of ICS containing therapies. $20-30 \%$ of the patients who appeared overtreated according to COPD recommendations had current asthma, which may explain the use of ICS. Symptomatic patients with mMRC of 2 or more and those with frequent exacerbations appeared undertreated irrespective of eosinophil counts. For patients initiating therapy (cohort 2), many patients in groups A, B and C appeared to be overtreated, although again $20 \%$ of the patients who appeared over-treated had current asthma, some patients in groups C \& D were undertreated and some in group D were overtreated having been started on triple therapy as their initial therapy (Table 4).

\subsection{Comorbid Conditions}

Diabetes, cardiovascular disease and osteoporosis were the most common comorbidities in patients in all GOLD groups in both cohorts 


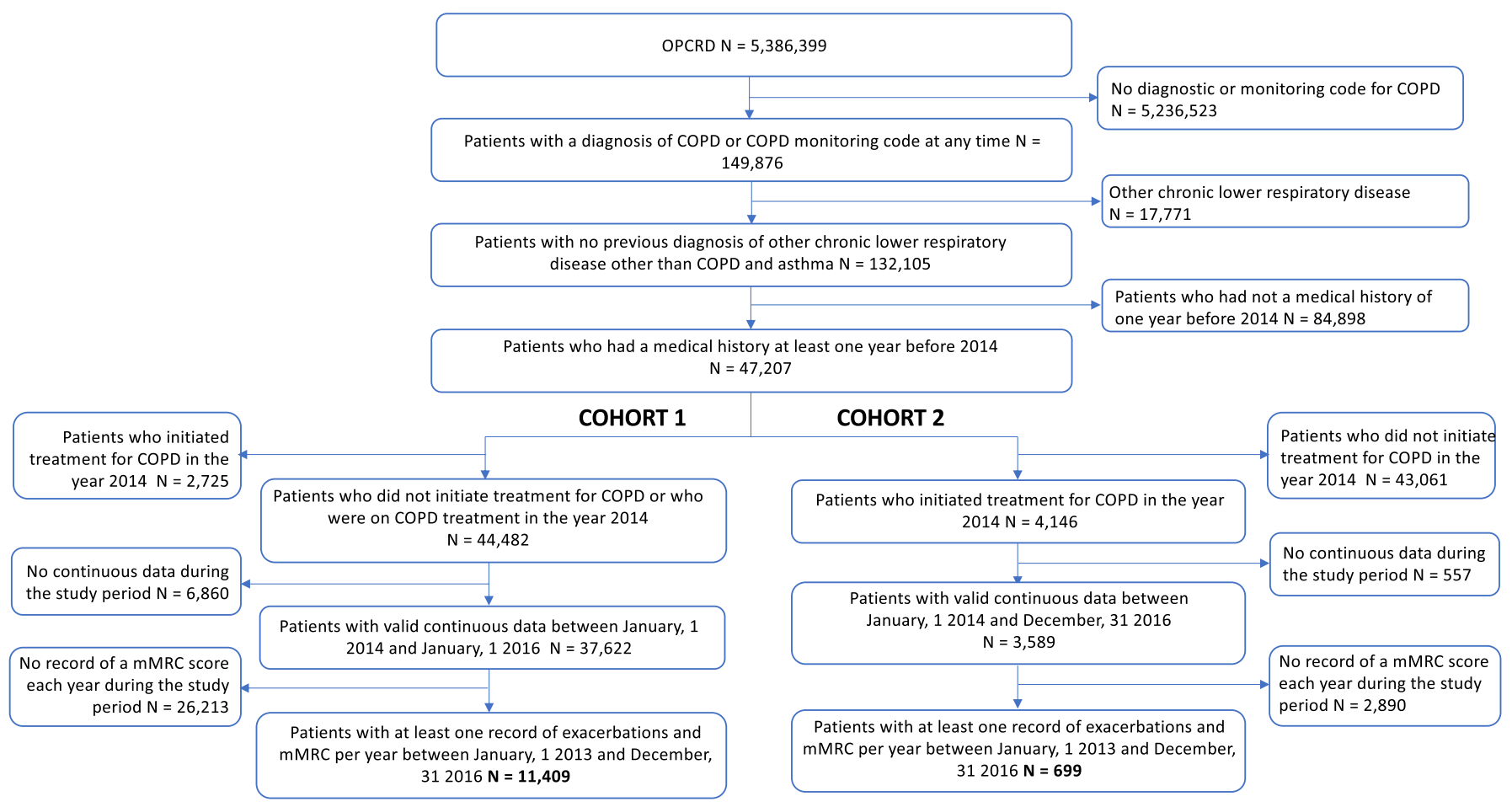

Fig. 1. Patient selection.

(Table 5 \& Appendix, tables S5 \& S6). There was no difference in the prevalence of comorbidities between the two cohorts of COPD patients, except for osteoporosis which was less common in patients initiating therapy (4.3\% vs. $7.7 \%$; OR: 0.54 (95\%CI $0.37-0.78$ ) $\mathrm{p}=0.001$ ) (Table 5).

Most comorbidities were more common in the established COPD patients than in patients who did not have COPD matched for age, sex and smoking history (Appendix, table S7), whereas cardiovascular comorbidities, anxiety/depression, rhinitis and gastroesophageal reflux were more common in COPD patients starting maintenance therapy than in their matched controls (Appendix, table S7).

\subsection{Exacerbations}

The mean exacerbation rates during follow-up for patients in each GOLD group for established patients and those initiating therapy is shown in Table 6. For the established patients (cohort 1) the mean rates for patients in groups $A$ and $B$ were higher and in groups $C$ and $D$ were lower during follow-up than in the year prior to ID (Table 1). Patients in GOLD groups B, C \& D had significantly higher exacerbation rates during follow-up than patients in group A (RRs: 1.42-3.69) (Table 6).

For patients initiating maintenance therapy (cohort 2) exacerbation rates were higher during follow-up than in the year prior to ID for patients in groups A \& B, but lower during follow up for patients in groups $C \& D$ (Table 1 ). Patients initiating therapy in GOLD groups $B, C \& D$ again had significantly higher exacerbation rates during follow-up than patients in group A (RRs: 1.60-2.90) (Table 6). The distribution and mean exacerbation rate per year during the follow up period of COPD patients initiating therapy in each GOLD group, categorised by appropriateness of therapy according to the 2019 GOLD Report, are shown in Appendix table S9. Exacerbation rates appeared slightly higher in people who were categorised as over-treated in groups A, B \& C and also higher in those that appeared under-treated in groups $C \& D$ but the numbers of patients in each category were small and the differences were not tested statistically.

\subsection{Pneumonia}

Pneumonia in the year prior to ID was significantly more commonly recorded in established patients with COPD than in matched controls, occurring in $1.1 \%$ of COPD patients and in $0.5 \%$ of controls (OR: $2.2095 \%$ CI $1.77-2.75$ ), and in $1.3 \%$ of patients starting maintenance therapy compared with $0.1 \%$ of matched controls (OR: 17.98 95\%Cl 3.89-83.18). In established COPD patients the proportion of patients in each GOLD group diagnosed with pneumonia during follow-up was $1.5 \%$ in group $A$ and $3.2-3.3 \%$ in groups B-D. However, no statistical differences between the GOLD groups and the rate of pneumonia was observed (data not shown).

\subsection{Escalation/De-escalation of Therapy}

Fig. 3 shows the proportion of patients initiating therapy (cohort 2) who had their therapy escalated, de-escalated, switched or left unchanged over the two years of follow-up by initial GOLD group. Patients in group B were slightly more likely to have their treatment switched or escalated than patients in other groups (table S10).

Appendix table S11 shows the appropriateness of maintenance therapy after two years, assessed according to the recommendations for initial therapy for the GOLD group in 2014. In patients in group A there was an equal tendency both to increase treatment in approximately a quarter of those appropriately treated at baseline, and de-escalate in those over treated at baseline. A similar pattern was seen in group B, but $11 \%$ of patients who were over-treated at baseline were de-escalated to therapy that appeared to leave them under-treated. In group $C$ half of the patients under-treated at baseline remained under-treated at follow-up, whilst most who were initially over-treated appeared appropriately treated at follow-up. In group D patients who were under-treated initially were either appropriately treated at follow-up (43\%) or over-treated (57\%), most who were appropriately or over-treated remained so.

\section{Discussion}

This study shows that nearly half of the COPD patients initiating therapy were in GOLD group A but nearly 10\% were in GOLD group D. 

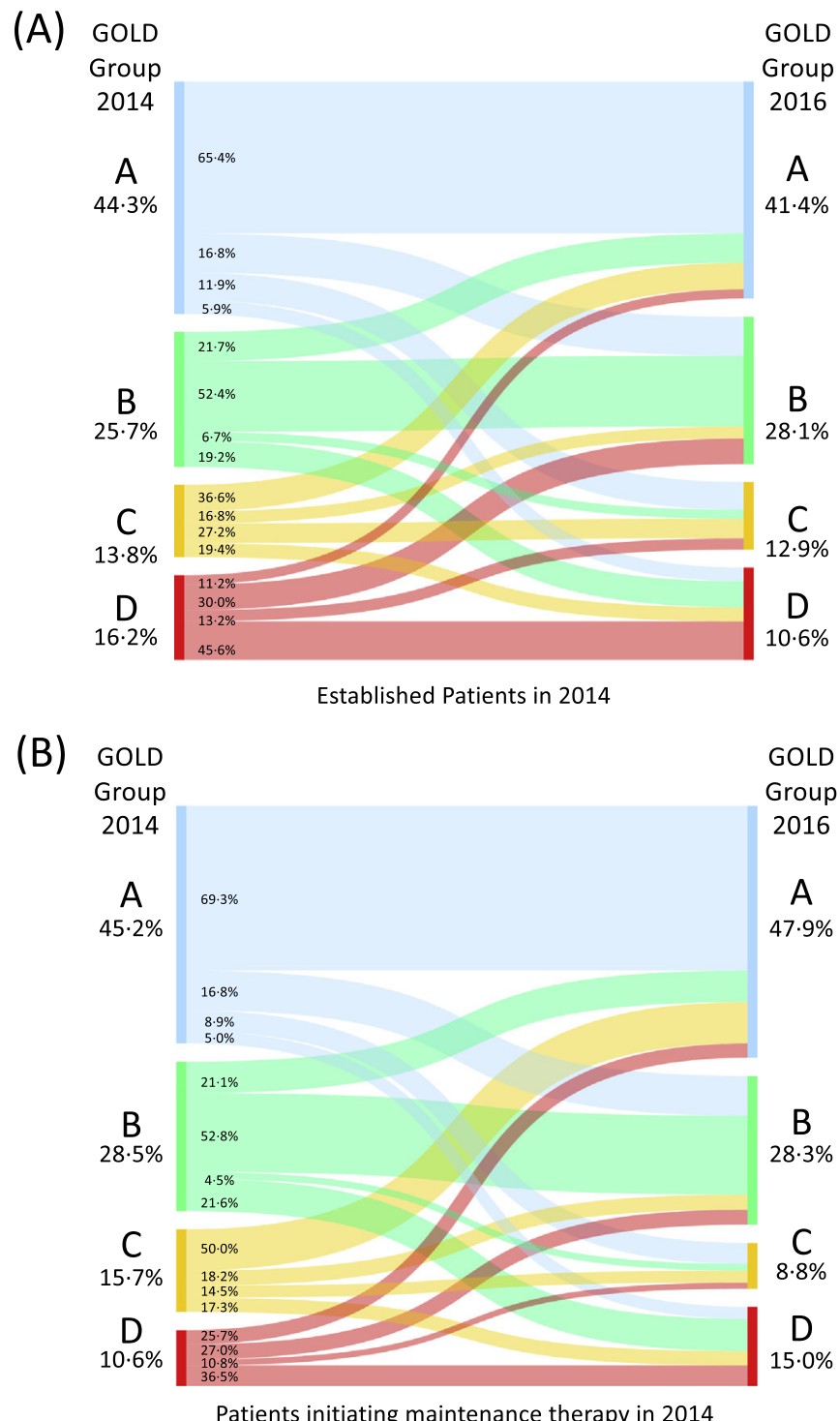

Fig. 2. Alluvial diagrams showing movement of patients between GOLD groups between 2014 and 2016 (A) Established COPD patients (B) COPD Patients initiating therapy.
The distribution of established patients among the groups was similar to that of the patients initiating therapy. The baseline demographic and disease characteristics of the patients in our study are similar to those previously reported for patients in the UK and internationally $[3,6]$. The fact that nearly half of patients initiating maintenance therapy were current smokers is concerning, considering that smoking cessation is the single most important intervention for all COPD patients. In both cohorts there was a striking similarity in the distribution of lung function impairment across the GOLD groups, with GOLD stage 2 being the most common abnormality. As would be expected there was a trend towards worse lung function in patients in the more symptomatic groups B \& D, but it was surprising that there were still more patients in these groups in both cohorts that had an $\mathrm{FEV}_{1} \geq 80 \%$ predicted (i.e. GOLD stage 1 ) than those with an $\mathrm{FEV}_{1}<30 \%$ predicted (i.e. GOLD stage 4 ).

Comorbidities were common in both cohorts and were more common in the cohorts than matched controls who did not have COPD. Nearly $20 \%$ of established patients and over $10 \%$ of patients initiating therapy had a diagnosis of current asthma. Apart from osteoporosis, comorbidities were not more common in patients with established COPD disease than in those initiating therapy. This may suggests that most comorbidities share common risk factors with COPD rather than being a consequence of having developed COPD, but the prevalence of osteoporosis may have been increased both by the likely more longstanding inactivity of established patients, systemic effects of COPD and steroid therapy [14]. Anxiety or depression were recorded as being present in approximately 1 in 5 patients with established COPD and in a similar proportion of patients initiating maintenance therapy. The recognition and management of these comorbidities is important as they are associated with increased levels of symptoms, a greater likelihood of being hospitalised, poor compliance with therapy and less success with smoking cessation strategies [15-18].

Assessing patients using the GOLD groups did identify patients at greater risk of exacerbations in both cohorts. The risk patients in group D compared to group A was greater in the established patients than in those initiating therapy. This probably reflects the fact that established patients were assessed as being in GOLD group D (i.e. having frequent exacerbations) while already on therapy, whereas patients initiating therapy may had greater opportunity for treatment to reduce the risk of exacerbations.

In both cohorts there was considerable change in symptoms and risk of exacerbations over two years, leading to potential reclassification of patients. However, in both cohorts nearly two thirds of patients in group A and half of the patients in group B remained in the same group. Group C was the least stable with only $27.2 \%$ of established

Table 3

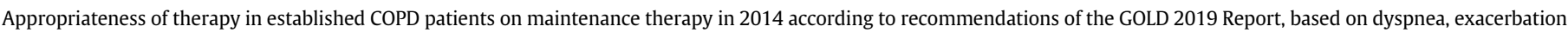
frequency and blood eosinophil counts at ID (cohort 1 ). (To avoid unintentional disclosure and to preserve confidentiality, cell counts $<5$ are reported as $<5$.

\begin{tabular}{|c|c|c|c|c|}
\hline \multirow[t]{3}{*}{ Treatment according to Gold 2019 report } & \multicolumn{4}{|c|}{ Baseline Characteristics } \\
\hline & \multicolumn{2}{|l|}{$\mathrm{mMRC}<2$} & \multicolumn{2}{|l|}{$\mathrm{mMRC} \geq 2$} \\
\hline & $<2$ exacerbations & $\geq 2$ exacerbations & $<2$ exacerbations & $\geq 2$ exacerbations \\
\hline Eos $<100$ & $N=290$ & $\mathrm{~N}=93$ & $\mathrm{~N}=197$ & $N=96$ \\
\hline Under-treatment (\%) & $0(0)$ & $36(38.7)$ & $77(39 \cdot 1)$ & $27(28 \cdot 1)$ \\
\hline Appropriate treatment (\%) & $159(54.8)$ & $0(0)$ & $5(2.5)$ & $0(0)$ \\
\hline Over-treatment $(\%)$ & $131(45 \cdot 2)$ & $57(61 \cdot 3)$ & $115(58.4)$ & $69(71.9)$ \\
\hline Number of overtreated patients with coexistent asthma (\%) & $24(18 \cdot 3)$ & $16(28 \cdot 1)$ & $25(21.7)$ & $15(21.7)$ \\
\hline Eos $100-300$ & $\mathrm{~N}=2862$ & $\mathrm{~N}=893$ & $\mathrm{~N}=1714$ & $\mathrm{~N}=1040$ \\
\hline Under-treatment (\%) & $0(0)$ & $673(75.4)$ & $743(43.4)$ & $637(61.3)$ \\
\hline Appropriate treatment (\%) & $1479(51.7)$ & $220(24 \cdot 6)$ & $971(56 \cdot 6)$ & $403(38.7)$ \\
\hline Over-treatment (\%) & $1383(48 \cdot 3)$ & $0(0)$ & $0(0)$ & $0(0)$ \\
\hline Number of overtreated patients with coexistent asthma (\%) & $354(25 \cdot 6)$ & $0(0)$ & $0(0)$ & $0(0)$ \\
\hline Eos $>300$ & $\mathrm{~N}=1523$ & $N=520$ & $N=891$ & $\mathrm{~N}=640$ \\
\hline Under-treatment (\%) & $0(0)$ & $210(40.4)$ & $376(42 \cdot 2)$ & $369(57 \cdot 7)$ \\
\hline Appropriate treatment (\%) & $691(45 \cdot 4)$ & $310(59.6)$ & $515(57.8)$ & $271(42 \cdot 3)$ \\
\hline Over-treatment (\%) & $832(54 \cdot 6)$ & $0(0)$ & $0(0)$ & $0(0)$ \\
\hline Number of overtreated patients with coexistent asthma (\%) & $233(28.0)$ & $0(0)$ & $0(0)$ & $0(0)$ \\
\hline Missing Eos (\% of group)) & $\mathrm{N}=378(7.5)$ & $\mathrm{N}=67(4.3)$ & $\mathrm{N}=135(4 \cdot 6)$ & $\mathrm{N}=68(3.7)$ \\
\hline
\end{tabular}


Table 4

Appropriateness of therapy prescribed in patients initiating maintenance therapy in 2014 according to recommendations for initial therapy in the GOLD 2019 Report (cohort 2 ). (To avoid unintentional disclosure and to preserve confidentiality, cell counts $<5$ are reported as $<5$ ).

\begin{tabular}{|c|c|c|c|c|}
\hline & Group A & Group B & Group C & Group D \\
\hline & $N=316$ & $\mathrm{~N}=199$ & $N=110$ & $\mathrm{~N}=74$ \\
\hline Under-treatment (\%) & $0(0)$ & $0(0)$ & $22(20.0)$ & $7(9.5)$ \\
\hline Appropriate treatment (\%) & $174(55.1)$ & $107(53.8)$ & $44(40.0)$ & $57(77.0)$ \\
\hline Over-treatment (\%) & $142(44.9)$ & $92(46.2)$ & $44(40.0)$ & $10(13.5)$ \\
\hline Number of overtreated patients with coexistent asthma (\%) & $28(19.7)$ & $18(19.6)$ & $10(22.7)$ & $<5$ \\
\hline
\end{tabular}

patients and $14.5 \%$ of patients initiating maintenance therapy remaining in the group after 2 years whilst most moved into the low exacerbation risk groups $\mathrm{A}$ and $\mathrm{B}$. One third of patients initiating therapy remained in GOLD group D after 2 years, but many patients moved to a lower exacerbation risk group, possibly as a result of effective management. Similar changes have been reported in the Eclipse cohort which predominantly contained established patients attending outpatient clinics in secondary care [19].

The therapy initiated in patients in groups A, B \& C differed from that currently recommended in the GOLD report [2] and from the therapy recommended by GOLD at the time [20]. At the time that these patients were being managed, clinicians in the UK were increasingly being encouraged by payors to use the GOLD recommendations rather than following the NICE guidelines as the latter had not been updated since 2010 [21]; however, not all clinicians would have been aware of the GOLD recommendations or encouraged to follow them. Approximately $20 \%$ of patients assessed as being overtreated had asthma as well as COPD and in these patients the use of ICS would have been justified and therefore they were appropriately treated but this still leaves a significant number of patients who were overtreated, predominantly with ICS. Despite these caveats, comparison of the therapy prescribed with the current GOLD recommendations and its correlation with exacerbation rates is of interest. The 2019 GOLD Report emphasises that the assessment of symptoms and risk of exacerbations originally should only be used as a basis for determining initial therapy and is not designed for reassessing patients per GOLD group during follow-up.

In our analysis, a substantial proportion of patients initiating therapy in groups A \& B were over-treated and $20 \%$ of patients in group C were under-treated. By definition COPD patients in this cohort of initiators all received maintenance therapy, so we cannot comment on the prevalence of patients with COPD who were experiencing symptoms or had a history of exacerbations but who did not receive any treatment. However, among the established COPD patients there appeared to be under-treatment of symptomatic patients and those continuing to exacerbate suggesting these factors were not being considered when patients were reviewed, and over-treatment of people with low eosinophil counts. At the time these patients were being treated the blood esosinophil count had not been recommended as a factor to guide maintenance therapy, but these results suggest that if it had been used as GOLD now recommend when reviewing therapy, it may have been possible to de-escalate therapy in a large number of patients. People with low levels of symptoms or low exacerbation rates also appeared over-treated but may have actually been successfully treated.

Over $40 \%$ of patients initiating maintenance therapy in groups A and B were prescribed an ICS containing therapies, with substantial initiation of ICS monotherapy. Our findings are in line with previous studies analysing adherence to recommendations on the management of COPD, although most of these looked at the appropriateness of therapy in patients classified into groups A-D whilst on therapy rather than their GOLD group at initiation of therapy [3-6]. The adherence to guideline recommendations in this study may represent a 'best case' scenario given the fact that considerable rigour had been used to diagnose COPD using spirometry and the likelihood that this would translate to considered therapy choices. Previous studies have examined the possible reasons underlying the difference between real-life practice and evidence-based treatment recommendations. Poor familiarity has been found to be associated with nonadherence to the previous recommendations on ICS and long-acting bronchodilators [22], and primary care physicians who had difficulty assessing response to therapy were less likely to adhere to recommendations on long-acting bronchodilator use [23]. However, in our analysis the mean exacerbation rate per year appeared higher in people who were categorised as over-treated in groups A, B \& C. Similar patterns have been reported in an analysis of prescribing at a Veterans Administration Medical Center in the USA [24]. This may suggest that additional clinical features associated with an increased risk of exacerbations led the managing clinicians to prescribe therapy which was at variance with the GOLD recommendations, but perhaps appropriate for that individual.

There were changes in therapy in the patients initiating maintenance therapy over the two years of follow-up, but most commonly treatment was left unchanged and for patients in groups A and B this meant that many remained over-treated. There was evidence of

\section{Table 5}

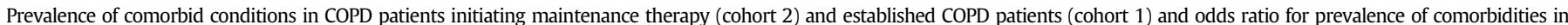
cohort 2 vs. cohort 1 . *Active at the index date, (To avoid unintentional disclosure and to preserve confidentiality, cell counts $<5$ are reported as $<5$.

\begin{tabular}{|c|c|c|c|c|}
\hline \multirow[t]{2}{*}{$\begin{array}{l}\text { Co-morbidity ever or in the year before the index } \\
\text { date, } n(\%)\end{array}$} & \multirow{2}{*}{$\begin{array}{l}\text { COPD patients initiating therapy } \\
\text { (Cohort 2) } \\
\mathrm{N}=699\end{array}$} & \multirow{2}{*}{$\begin{array}{l}\text { Established COPD patients } \\
\text { (Cohort } 1 \text { ) } \\
\mathrm{N}=11,409\end{array}$} & \multirow[t]{2}{*}{$\begin{array}{l}\text { OR } 95 \%(\mathrm{CI}) \\
\text { Cohort } 2 \text { vs. Cohort } 1\end{array}$} & \multirow[t]{2}{*}{ p-Value } \\
\hline & & & & \\
\hline Diabetes mellitus & $103(14 \cdot 7)$ & $1801(15 \cdot 8)$ & $0.92(0.74-1.14)$ & 0.46 \\
\hline Hypertension & $303(43.4)$ & $5359(47.0)$ & $0.86(0.74-1.01)$ & 0.06 \\
\hline Cardiovascular disease* & $71(10 \cdot 2)$ & $1216(10 \cdot 7)$ & $0.95(0.74-1.22)$ & 0.68 \\
\hline Heart failure* & $12(1 \cdot 7)$ & $162(1.4)$ & $1.21(0.67-2 \cdot 19)$ & 0.52 \\
\hline Ischaemic heart disease & $128(18 \cdot 3)$ & $2154(18.9)$ & $0.96(0.79-1.17)$ & 0.71 \\
\hline Sleep apnoea & $<5$ & $43(0.4)$ & $0.38(0.05-2.75)$ & 0.34 \\
\hline Nasal polyps & $<5$ & $24(0.2)$ & $1.36(0.32-5.77)$ & 0.67 \\
\hline Osteoporosis & $30(4 \cdot 3)$ & $874(7.7)$ & $0.54(0.37-0.78)$ & 0.001 \\
\hline Chronic Kidney Disease Stage IV-V & $14(2 \cdot 0)$ & $247(2 \cdot 2)$ & $0.92(0.54-1.59)$ & 0.77 \\
\hline Anxiety/Depression* & $136(19.5)$ & $2326(20.4)$ & $0.94(0.78-1.14)$ & 0.55 \\
\hline Eczema* & $22(3 \cdot 2)$ & $385(3.4)$ & $0.93(0.60-1.44)$ & 0.75 \\
\hline Rhinitis* & $48(6.9)$ & $903(7.9)$ & $0.86(0.64-1.16)$ & 0.32 \\
\hline Gastroesophageal reflux disease* & $92(13 \cdot 2)$ & $1763(15 \cdot 5)$ & $0.83(0.66-1.04)$ & $0 \cdot 10$ \\
\hline
\end{tabular}


Table 6

Mean annual exacerbation rates over 2 years in established COPD patients and those initiating maintenance therapy in 2014 by GOLD group at baseline. SD = standard deviation; $\mathrm{RR}=$ rate ratio; $\mathrm{CI}=$ confidence interval.

\begin{tabular}{|c|c|c|c|c|}
\hline \multirow{2}{*}{$\begin{array}{l}\text { GOLD group } \\
\text { in } 2014\end{array}$} & \multicolumn{4}{|c|}{ Exacerbation rate per year per GOLD group } \\
\hline & $\mathrm{N}$ & $\begin{array}{l}\text { Mean } \\
(\mathrm{SD})\end{array}$ & $\mathrm{RR}_{\text {crude }}(95 \% \mathrm{CI})$ & $\begin{array}{l}\mathrm{RR}_{\text {age and sex-adjusted }}(95 \% \\
\mathrm{CI})\end{array}$ \\
\hline \multicolumn{5}{|c|}{ Established COPD patients (Cohort 1) } \\
\hline Group A & 5053 & $0.7(0.9)$ & Reference & Reference \\
\hline Group B & 2938 & $0.9(1.0)$ & $1.41(1.34-1.48)$ & $1.42(1.34-1.49)$ \\
\hline Group C & 1574 & $1.9(1.7)$ & $2.89(2.73-3.06)$ & $2.87(2.71-3.04)$ \\
\hline Group D & 1844 & $2.4(1.9)$ & $3.71(3.52-3.91)$ & $3.69(3.50-3.89)$ \\
\hline \multicolumn{5}{|c|}{ COPD patients initiating therapy (Cohort 2) } \\
\hline Group A & 316 & $0.6(0.8)$ & Reference & Reference \\
\hline Group B & 199 & $1.0(1.2)$ & $1.61(1.30-1.99)$ & $1.60(1.29-1.97)$ \\
\hline Group C & 110 & $1.5(1.6)$ & $2.68(2.11-3.40)$ & $2.68(2 \cdot 11-3.41)$ \\
\hline Group D & 74 & $1.8(1.9)$ & $2.93(2.24-3.84)$ & $2.90(2.21-3.81)$ \\
\hline
\end{tabular}

escalation of therapy in line with recommendations in patients considered under-treated initially in groups $C$ and D, suggesting that clinicians had reviewed the patients and adjusted therapy based on continued symptoms or exacerbations. It was not possible to assess the appropriateness of changes in in established COPD patients according to the recommendations for follow-up in the GOLD 2019 Report as information was not available in the database about individual's treatable traits at the time of review.

\subsection{Strengths and Limitations of the Study}

Our study has several strengths including the large sample size, completeness of follow-up and the robustness of the COPD diagnosis in OPCRD which is based on symptoms and spirometry. OPCRD is a well-maintained database which is used frequently for observational research and it contains information which is prospectively collected, and not subjected to recall bias. The dataset draws from a geographically and socioeconomically diverse population, and therefore we believe that the study results reflect routine clinical practice in the UK and show the true distribution of UK patients among the GOLD groups, and their demographic and disease characteristics. It also shows the differences between real-world prescribing patterns in the UK and guideline recommendations.

Potential study limitations include the fact that the data set reflects the demographics of patients and clinical practice in the UK only. Furthermore, to be included in the study patients had to have an MMRC score and the number of exacerbations recorded in their medical notes in 2014, 2015 and 2016 and may not therefore be fully representative of all patients with COPD. Different guidelines, such as the NICE COPD guideline may have influenced management and availability of medication and initiatives by payors may also have influenced prescribing, for example inhalers containing LABA/ LAMA were only launched in the UK in late 2014 [25]. Patients' classification into the GOLD groups was based solely on their mMRC scores as these are routinely recorded in UK primary care practice; however, some clinicians may have used CAT scores to assess patients and make treatment decisions. CAT scores are not systematically recorded in all patients. It is also not possible to determine if patients were adherent with their therapy and thus the presence of symptoms or occurrence of exacerbations in patients on treatment may reflect under-treatment or poor adherence to prescribed medications, or both.

In summary, this analysis indicates that the majority of patients are not at high risk of exacerbations and that smoking and comorbidities are common at the time patients initiate maintenance therapy. Many patients in GOLD groups A and B are over-treated when compared to the GOLD recommendations, in particular they are prescribed ICS alone or in combination with a LABA as their first maintenance therapy. Many patients remained symptomatic and continued to have exacerbations despite treatment, but there was evidence that therapies were escalated and de-escalated in response to this; however many remained overtreated and continued work is needed to improve clinicians assessment and management of patients with COPD using dissemination methods

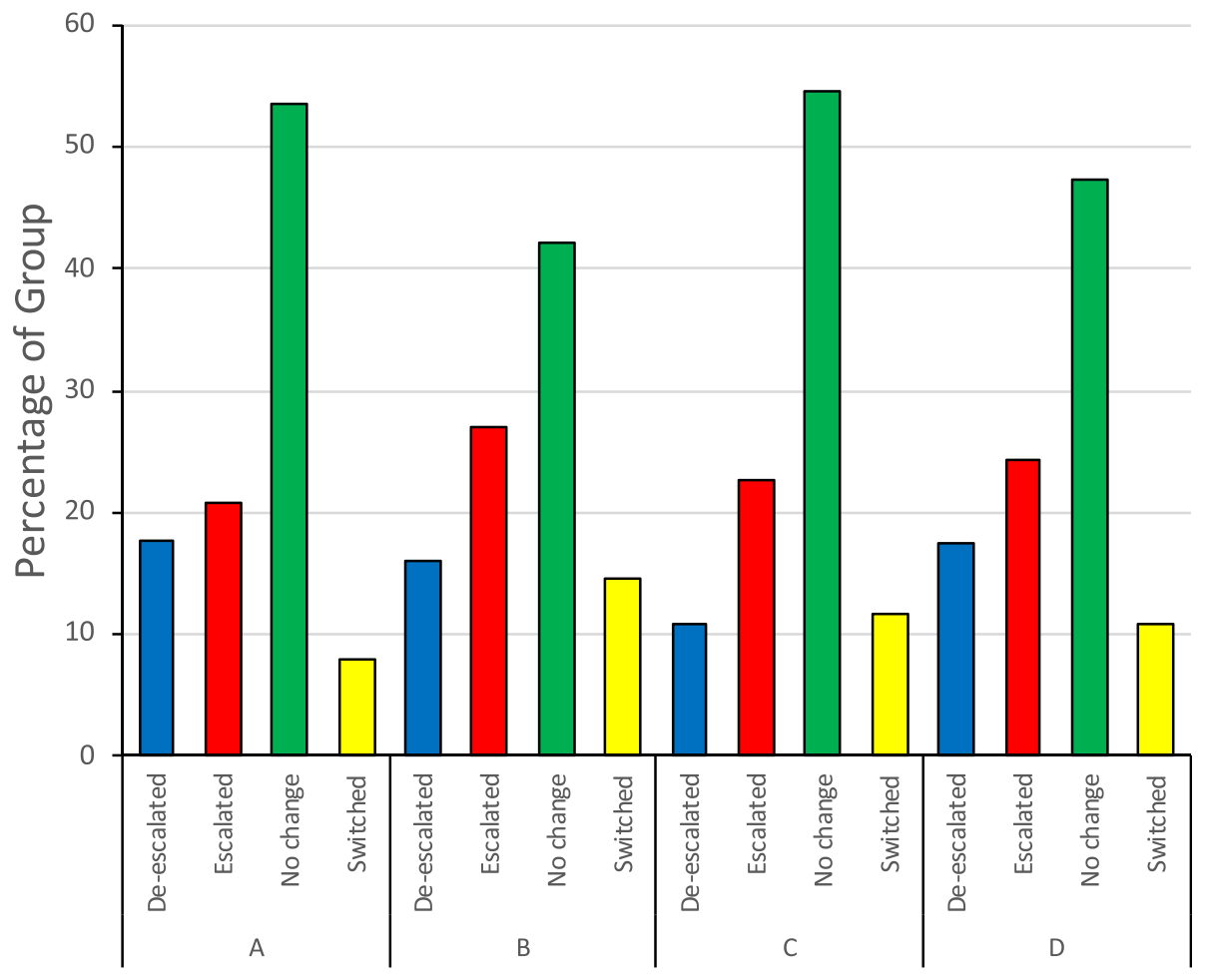

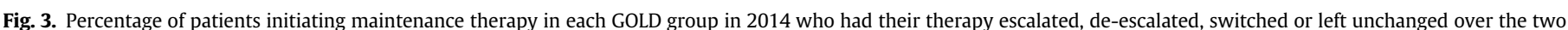
years of follow-up. 
more likely to change behaviour [26] in order to limit the risks and costs associated with under-treatment and over-treatment and to improve patient outcomes.

\section{Authors' Contributions}

The authors meet criteria for authorship as recommended by the International Committee of Medical Journal Editors, take responsibility for the integrity of the work as a whole, contributed to the writing and reviewing of the manuscript, and have given final approval for the version to be published. All authors had full access to the data in this study and take complete responsibility for the integrity of the data and accuracy of the data analysis. D Halpin, $\mathrm{H}$ de Jong, V Carter and David Price were involved in the conception/design of the study and analysis/interpretation of data. D Skinner was involved in acquisition and analysis of the data.

\section{Funding}

Observational and Pragmatic Research Institute (OPRI).

\section{Acknowledgements/Funding}

This work was funded by OPRI with no external contributions.

\section{Declaration of Competing Interest}

Prof. Halpin reports personal fees from AstraZeneca, personal fees and non-financial support from Boehringer Ingelheim, personal fees from Chiesi, personal fees and non-financial support from GlaxoSmithKline, personal fees and non-financial support from Novartis, personal fees from Pfizer, outside the submitted work. He is also a member of the GOLD Board of Directors and Science Committee. Dr. de Jong, Ms. Carter \& Dr. Skinner are employed by the Observational and Pragmatic Research Institute Pte Ltd. (OPRI). Prof. Price reports grants from AKL Research and Development Ltd., personal fees from Amgen, grants and personal fees from AstraZeneca, grants and personal fees from Boehringer Ingelheim, grants from British Lung Foundation, grants and personal fees from Chiesi, grants and personal fees from Circassia, personal fees from Cipla, personal fees from GlaxoSmithKline, personal fees from Kyorin, personal fees from Merck, grants and personal fees from Mylan, grants and personal fees from Mundipharma, grants and personal fees from Napp, grants and personal fees from Novartis, grants and personal fees from Pfizer, grants and personal fees from Regeneron Pharmaceuticals, grants from Respiratory Effectiveness Group, grants and personal fees from Sanofi Genzyme, grants and personal fees from Teva, grants and personal fees from Theravance, grants from UK National Health Service, grants and personal fees from Zentiva (Sanofi Generics), non-financial support from Efficacy and Mechanism Evaluation programme, non-financial support from Health Technology Assessment, outside the submitted work; and stock/stock options from AKL Research and Development Ltd. which produces phytopharmaceuticals; and owns $74 \%$ of the social enterprise Optimum Patient Care Ltd. (Australia and UK) and 74\% of Observational and Pragmatic Research Institute Pte Ltd. (Singapore).

\section{Appendix A}

\section{A.1. Data Requirements and Data Extracted From OPCRD}

To estimate the patient characteristics in each cohort, and to prevent user bias in the study of patients in cohort 2, every patient had to be registered with a general practitioner (GP) for at least one year before the ID. Patients had to have had continuous medical record data between January 1, 2014 and December 31, 2016, to have had at least one record of a modified MRC (mMRC) dyspnoea score per year between January 1, 2014 and December 31, 2016, and to have had at least one record of exacerbation frequency (including none) per year between January 1, 2013 and December 31, 2015. Patients were excluded if they had a previous diagnosis of other lower respiratory disease other than COPD, asthma, or bronchitis at any time.

For eligible patients the following data were extracted from the OPCRD: age at ID; sex; body mass index (BMI, $\mathrm{kg} / \mathrm{m} 2$ ) within 5 years prior to ID; smoking status (categorised as never smokers, current smokers, former smokers); $\mathrm{FEV}_{1} \%$ predicted (categorised by GOLD stage: GOLD 1: $\geq 80$, GOLD 2: 50-79, GOLD 3: 30-49, GOLD 4: <30); mMRC dyspnoea score (categorised as: $0-1$ and $\geq 2$ ); blood eosinophil count (categorised as $\geq 0.30 \times 10^{9} / \mathrm{L}$ (high) and $<0.30 \times 10^{9} / \mathrm{L}($ low)); prescription of respiratory drugs; the presence of comorbidities recorded as active problems in the year prior to the ID; and whether or not patients had a diagnosis of pneumonia in the year prior to the ID. Based on their mMRC score and history of exacerbations in the previous year, patients within each cohort were then classified by the GOLD 2017 classification scheme as GOLD group A, B, C or D in the year 2014 and 2016 [1].

COPD exacerbations were defined as the occurrence of the following events:

a. Unscheduled hospital admission with acute COPD exacerbation as the primary diagnosis AND/OR,

b. Unscheduled COPD-related accident \& emergency (A\&E) attendance AND/OR,

c. An acute course of oral corticosteroid AND/OR.

d. Antibiotics prescribed with evidence of a lower respiratory consultation.

When oral corticosteroids and antibiotics were prescribed during a routine respiratory review (mMRC and a prescription of oral corticosteroids and/or antibiotics at the same day) the prescription of both of these drugs was not be included in the algorithm for estimating the number of exacerbations. An exacerbation within 14 days from a previous one was considered to belong to the same event.

\section{A.2. Complementary Analysis on Comorbidities}

We compared the comorbidities of the patients in cohort 2 with the ones from cohort 1 by conducting a logistic regression to derive odds ratios (ORs) and $95 \%$ CIs. We also examined the prevalence of comorbid conditions in a general population to determine whether the overall prevalence of comorbidities was higher in patients with established COPD. For each patient with COPD we randomly selected up to four controls matched by age ( \pm 2 years), sex and smoking status (never/ever/ex-smoker), and randomly selected from the population of patients at risk for COPD at the index date of the patient with COPD. We then assessed the association between the prevalence of comorbid conditions and having COPD using conditional logistic regression.

\section{Appendix B. Supplementary Data}

Supplementary data to this article can be found online at https:// doi.org/10.1016/j.eclinm.2019.07.003.

\section{References}

[1] Vogelmeier CF, Criner GJ, Martinez FJ, et al. Global strategy for the diagnosis, management, and prevention of chronic obstructive lung disease 2017 report: GOLD executive summary. Am J Respir Crit Care Med 2017;195(5):557-82.

[2] Global Initiative for Chronic Obstructive Lung Disease (GOLD). Global strategy for the diagnosis, management, and prevention of chronic obstructive pulmonary disease. 2019 report. Available from: http://www.goldcopd.org/; 2018.

[3] Price D, West D, Brusselle G, et al. Management of COPD in the UK primary-care setting: an analysis of real-life prescribing patterns. Int J Chron Obstruct Pulmon Dis 2014;9:889-905 [Epub 2014/09/12]. 
[4] Corrado A, Rossi A. How far is real life from COPD therapy guidelines? An Italian observational study. Respir Med 2012;106(7):989-97 [Epub 2012/04/10].

[5] Palmiotti GA, Lacedonia D, Liotino V, et al. Adherence to GOLD guidelines in reallife COPD management in the Puglia region of Italy. Int J Chron Obstruct Pulmon Dis 2018;13:2455-62.

[6] Ding B, Small M, Holmgren U. A cross-sectional survey of current treatment and symptom burden of patients with COPD consulting for routine care according to GOLD 2014 classifications. Int J Chron Obstruct Pulmon Dis 2017;12:1527-37.

[7] Roche N, Lepage T, Bourcereau J, Terrioux P. Guidelines versus clinical practice in the treatment of chronic obstructive pulmonary disease. Eur Respir J 2001;18:903-8.

[8] Jochmann A, Scherr A, Jochmann DC, et al. Impact of adherence to the GOLD guidelines on symptom prevalence, lung function decline and exacerbation rate in the Swiss COPD cohort. Swiss Med Wkly 2012;142:w13567 [Epub 2012/04/07].

[9] Drivenes E, Ostrem A, Melbye H. Predictors of ICS/LABA prescribing in COPD patients: a study from general practice. BMC Fam Pract 2014;15:42. [Epub 2014/03/07].

[10] Bourbeau J, Sebaldt RJ, Day A, et al. Practice patterns in the management of chronic obstructive pulmonary disease in primary practice: the CAGE study. Can Respir J 2008;15(1):13-9.

[11] Roche N, Pribil C, Van Ganse E, et al. Real-life use of fluticasone propionate/salmeterol in patients with chronic obstructive pulmonary disease: a French observational study. BMC Pulm Med 2014;14:56. [Epub 2014/04/04].

[12] Landis JR, Koch GG. The measurement of observer agreement for categorical data. Biometrics 1977;33(1):159-74 [Epub 1977/03/01].

[13] Halpin DMG, De Jong H, Carter V, Skinner D, Price D. Prevalence of comorbidities in established and newly treated patients with COPD according to GOLD 2017 in a UK primary care population. Eur Resp J 2018;52:PA3616.

[14] Romme EA, Smeenk FW, Rutten EP, Wouters EF. Osteoporosis in chronic obstructive pulmonary disease. Expert Rev Respir Med 2013;7(4):397-410 [Epub 2013/08/21].

[15] Omachi TA, Katz PP, Yelin EH, et al. Depression and health-related quality of life in chronic obstructive pulmonary disease. Am J Med 2009;122(8):778. [e9-15].

[16] Jennings JH, Digiovine B, Obeid D, Frank C. The association between depressive symptoms and acute exacerbations of COPD. Lung 2009;187:128-35.
[17] Albrecht JS, Park Y, Hur P, et al. Adherence to maintenance medications among older adults with chronic obstructive pulmonary disease: the role of depression. Ann Am Thorac Soc 2016;13:1497-504.

[18] Halpin D, Hyland M, Blake S, et al. Understanding fear and anxiety in patients at the time of an exacerbation of chronic obstructive pulmonary disease: a qualitative study. JRSM Open 2015;6(12) [2054270415614543. Epub 2015/12/15].

[19] Faner R, Noell G, Badia JR, et al. Distribution, temporal stability and association with all-cause mortality of the 2017 GOLD groups in the ECLIPSE cohort. Respir Med 2018;141:14-9.

[20] Global Initiative for Chronic Obstructive Lung Disease (GOLD). Global strategy for the diagnosis, management, and prevention of chronic obstructive pulmonary disease: revised 2011. Available from http://www.goldcopd.org/uploads/users/ files/GOLD_Report_2011_Jan21.pdf; 2011.

[21] National Institute for Health and Clinical Excellence. Chronic obstructive pulmonary disease: Management of chronic obstructive pulmonary disease in adults in primary and secondary care. London: National Clinical Guideline Centre; 2010. Available from http://www.nice.org.uk/guidance/cg101/.

[22] Perez X, Wisnivesky JP, Lurslurchachai L, Kleinman LC, Kronish IM. Barriers to adherence to COPD guidelines among primary care providers. Respir Med 2012;106(3):374-81 [Epub 2011/10/18].

[23] Salinas GD, Williamson JC, Kalhan R, et al. Barriers to adherence to chronic obstructive pulmonary disease guidelines by primary care physicians. Int J Chron Obstruct Pulmon Dis 2011;6:171-9.

[24] Foda HD, Brehm A, Goldsteen K, Edelman NH. Inverse relationship between nonadherence to original GOLD treatment guidelines and exacerbations of COPD. Int J Chron Obstruct Pulmon Dis 2017;12:209-14 [Epub 2017/01/27].

[25] Lawrence J. Combination inhaler Anoro Ellipta launched for COPD. Pharm J 2014;293 [online].

[26] Halpin DMG. Understanding irrationality: the key to changing behaviours and improving management of respiratory diseases? Lancet Respir Med 2018;6(10): 737-9. 